\title{
Characterization of the beam scraping system of the CERN Super Proton Synchrotron
}

\author{
Alessio Mereghetti॰ ${ }^{*}$ and Francesco Cerutti \\ CERN, CH-1211 Geneva 23, Switzerland \\ Robert B. Appleby \\ School of Physics and Astronomy, The University of Manchester, Manchester M13 9PL, United Kingdom \\ and Cockcroft Institute, Daresbury Laboratory, Warrington WA4 4AD, United Kingdom
}

(Received 6 July 2020; revised 10 March 2021; accepted 15 March 2021; published 19 April 2021)

\begin{abstract}
The Super Proton Synchrotron at CERN is equipped with a scraping system for halo cleaning at beam transfer to the Large Hadron Collider. The system is composed of movable graphite blades mechanically swept through the beam to remove tails immediately before beam transfer. Due to the mechanical movement, beam particles are intercepted by a small volume of material with consequent concentration of energy deposition and high thermal loads. The blades were tested with beam to verify their resistance to the most extreme scraping conditions. Even though the beam was prematurely dumped by the beam loss monitoring system, the microstructural analysis of the blades following the test found signs of material sublimation. The test setup was reproduced in simulation to reconstruct the levels of energy deposition actually reached in the blades during the test; values are compatible with local material sublimation, in agreement with the microstructural analysis. Simulations were carried out by coupling the sixTrack tracking code, used for single particle beam dynamics in circular accelerators for high energy physics, to the FLUKA Monte Carlo code, for particle-matter interactions. The time evolution of the beam intensity measured during scraping and the distribution of losses around the ring were used for an extensive benchmark of the simulation tool against measurements taken during the test. This work presents the endurance test together with simulation results and the benchmark of the simulation tool. The quantitative agreement between simulations and measurements proves the quality of the analyses and the maturity of the simulation tool, which can be reliably used to predict the performance of cleaning systems in circular accelerators.
\end{abstract}

DOI: $10.1103 /$ PhysRevAccelBeams.24.043002

\section{INTRODUCTION}

Accelerator R\&D is nowadays vital to answer the quest for more energetic and brighter beams set by high energy physics investigations [1]. Technological advancements are important in all the systems involved in the operation of accelerators [2]. In particular, machine protection systems have recently become more and more important, as beams become increasingly destructive with intensities and energies [3], and devices become more and more expensive and fragile. In particular, the growing deployment of superconducting (SC) devices has tightened the requirements on safe machine operation.

\footnotetext{
*alessio.mereghetti@cern.ch
}

Published by the American Physical Society under the terms of the Creative Commons Attribution 4.0 International license. Further distribution of this work must maintain attribution to the author(s) and the published article's title, journal citation, and DOI.
Machine protection systems can reach high levels of complexity, involving different techniques of detecting equipment failures, sophisticated interlocks cross-checking different operational settings at the same time, and optimum design of beam-intercepting devices. The interaction of the lost particles with protection devices is the ultimately critical factor, as the beam characteristics may undermine the hardware integrity. Beam impact parameters, together with material properties, are the factors ruling damage mechanisms. The analysis of faults and loss scenarios and estimation of consequences on sensitive equipment through computer simulations are fundamental steps toward the design of a machine protection system that can ensure the safe operation of the accelerator. In order to have a sound prediction of the performance of beam-intercepting devices, the complexity of simulation tools is growing rapidly to cope with the more demanding requirements set by brighter and more energetic beams; the ultimate goal is to be confident about the endurance of the intercepting devices and nearby accelerator components in any foreseeable loss scenario. 
As an example, the Large Hadron Collider (LHC) [4] at CERN is the largest and most powerful hadron accelerator for high energy physics presently in operation. The LHC is a SC machine; hence, its magnets are sensitive to local beam losses, not only because of the possible damage induced by a direct beam impact but also because even tiny fractions of lost beam can heat the coils, potentially leading to a quench [5]. Recovery from quenches is a lengthy process, implying considerable machine downtime; thus, their occurrence must be minimized.

The LHC is equipped with a sophisticated collimation system [4], aimed at concentrating losses from the circulating beams in specific locations of the ring, and hence avoiding the risk of quenches. During injection, protection against miskicked beams is not granted by the LHC collimation system, but by a series of passive systems [6]. Furthermore, scrapers $[7,8]$ are used to regularly deplete tails on the transverse planes before injecting beam in the LHC. This system is installed in the Super Proton Synchrotron (SPS) [9], the last stage of acceleration in the LHC injection chain. Being far from SC magnets, the system can routinely remove transverse tails immediately before beam transfer with no risk of inducing quenches.

The SPS scrapers are movable graphite blades mechanically swept through the beam to remove tails immediately before beam transfer to the LHC. Due to the mechanical movement, beam protons are intercepted by a small volume of material with a concentration of energy deposition and high thermal loads. To prove the endurance of the blades, a test with beam in the most extreme scraping conditions was carried out in 2013. After the test, the blades were dismounted and a microstructural analysis was carried out by means of the scanning electron microscope (SEM), to reveal possible signs of damage.

An extensive simulation campaign was carried out to correlate the energy deposition reached during the test to the induced damage. Simulations were performed coupling $[10,11]$ sixTrack $[12,13]$, a symplectic tracking code for single particle beam dynamics in circular accelerators, to the FLUKA [14,15] Monte Carlo code, for describing the interaction of beam particles with intercepting devices. The asset of the FLUKA-SixTrack is the full deployment of the FLUKA geometry and physics module for particle-matter interactions, allowing users to simulate any arbitrarily complicated device, contrary to other state-of-art codes for estimating cleaning performance, which make substantial assumptions on geometry and material description of the intercepting devices.

This paper presents the endurance test of the SPS scraper blades, together with results from the simulation campaign. The hardware used in the test is presented first (Sec. II), with a brief introduction to the SPS, the scrapers and the monitors used during the test. Then, the endurance test and its main outcomes are presented (Sec. III). Even though beams were prematurely dumped by the beam loss monitoring (BLM) system, the microstructural analysis revealed signs of damage, compatible with local sublimation of graphite. Afterward, the paper reports the results of the simulation campaign (Sec. IV), which show that sublimation was reached during the test, in accordance with measurements. The endurance test of the SPS scraper blades was the first extensive application of the FLUKAsixTrack coupling; hence, the paper also presents a quantitative benchmark of simulation results against the measurements taken during the test. The good agreement between simulation results and measurements proves the quality of the analyses and the maturity of the simulation tool, which can be reliably used to predict the performance of cleaning systems in hadron circular accelerators at the frontier of high energy physics.

\section{THE HARDWARE USED IN THE TEST}

The SPS [9] is the last stage of acceleration before injection into the LHC [6]. It accelerates protons from 26 to $450 \mathrm{GeV} / c$, when they are transferred to the LHC via two dedicated transfer lines [6]. The SPS is a $7 \mathrm{~km}$-long synchrotron, structured in six identical arcs and long straight sections (LSSs). An LSS and half of the upstream and downstream arcs form a sextant. Each LSS is dedicated to one or more specific tasks; LSS1, which hosts the scrapers, is devoted to beam injection and dumping. Figure 1 shows the layout and optics of the LSS1.

Figure 2 shows an example of the SPS cycle for LHC beams [6]. At flat bottom, four consecutive injections take place, spaced by $3.6 \mathrm{~s}$; the energy ramp starts immediately after the last injection and accelerates the beam at a rate of up to $80 \mathrm{GeV} \mathrm{s}^{-1}$; at the flattop plateau, the extraction bumps are switched on and the extraction kickers fired; finally, magnets are ramped down to get ready to start with a new cycle. The total duration is $21.6 \mathrm{~s}$. Tail scraping is performed just before extraction toward the LHC to minimize tail repopulation. More in detail, it is performed not exactly at flattop, but at the end of the ramp, in order not to populate the abort gap. Scraping is visible in the beam current profile shown in Fig. 2 as the two tiny decreases toward the end of the ramp, which correspond to the removal of beam halo on the horizontal (first one) and the vertical (second one) planes, respectively. Almost the same amount of beam per plane is scraped away.

\section{A. The scrapers}

The SPS scrapers $[7,8]$ are movable graphite blades quickly swept through the beam for tail removal immediately before beam transfer to the LHC. The blades are $1 \mathrm{~cm}$ in length, and the graphite has a density of $1.83 \mathrm{~g} \mathrm{~cm}^{-3}$. Two blades are installed, one for each plane of cleaning. During injection in the SPS and for most of the ramp, the blades stay idle in the "parking" position (see Fig. 3, upper frame); just before scraping, they are moved to the "target" 


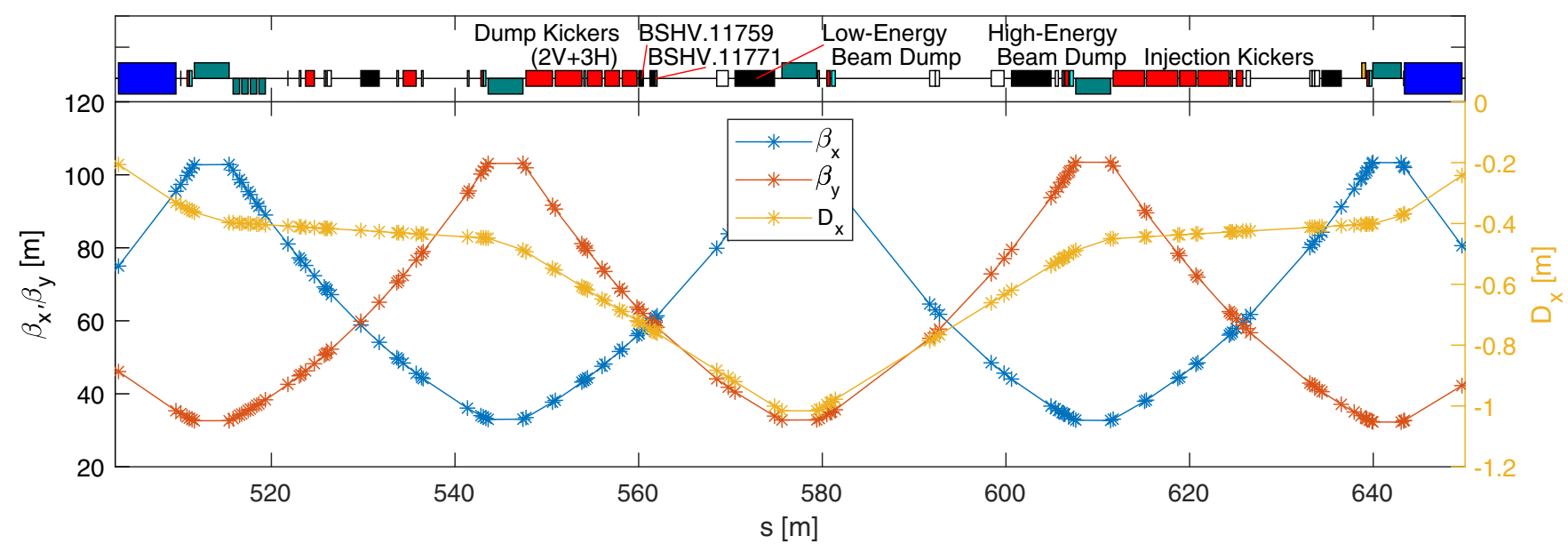

FIG. 1. Layout (upper frame) and linear optics functions (lower frame) of LSS1. Scrapers, injection and dump kickers, as well as dump absorbers are highlighted.

position, i.e., the desired position of scraping; once triggered, the blades are quickly swept through the beam, with a nominal speed of $80 \mathrm{~mm} \mathrm{rms}^{-1}$. Finally, the blades are moved back to the parking position, closing the mechanical cycle.

The SPS scrapers are installed in the LSS1 in two sets for redundancy reasons, close to each other to have similar optics functions and operational settings (see Fig. 1). Each set is equipped with a blade per plane and the necessary mechanics (see Fig. 3, lower frame). The two sets are named BSHV.11759 and BSHV.11771; the former, upstream of the other one, is kept as spare, whereas the latter is regularly used for operation.

Halo cleaning implies a decrease in beam current, and ultimately in instantaneous luminosity in the LHC at collision. Thus, an optimal scraping position was identified [7], i.e., $3.5 \sigma$, which assures for an effective tail removal with a negligible loss in luminosity. For a Gaussian beam

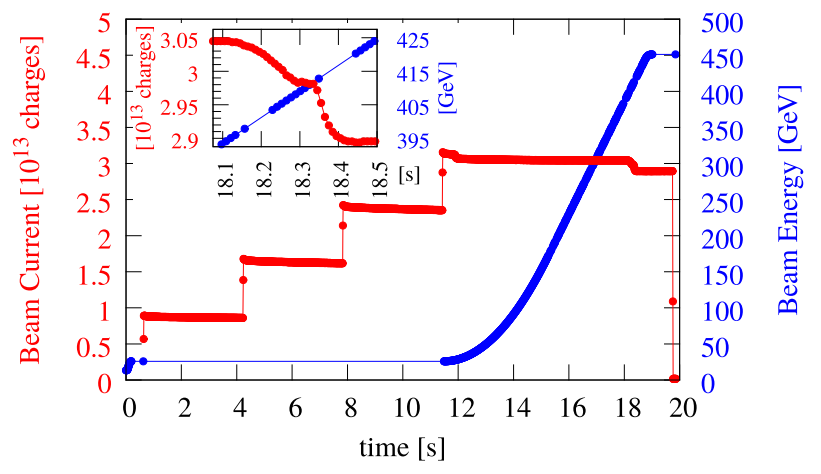

FIG. 2. Example of the SPS cycle for LHC beams: beam current (red curve, left vertical axis) and energy (blue curve, right vertical axis). It should be noted that the shown profile of beam current ends with a dump event and not with extraction; moreover, magnet ramp down is omitted. The inset shows the effect of both horizontal and vertical scrapings. profile, this target position corresponds to few percent of beam intensity. The same intensity reduction is achieved on both planes (see Fig. 2).

The SPS scrapers can also be used as a diagnostics tool to probe the beam transverse distribution via so-called "scraper scans." A scraper scan is a series of measurements where the amount of scraped beam is recorded as a function of the blade position. The scans are generally performed inwards, i.e., the scraping position is incrementally set closer to the beam center. During scraper scans, the scrapers
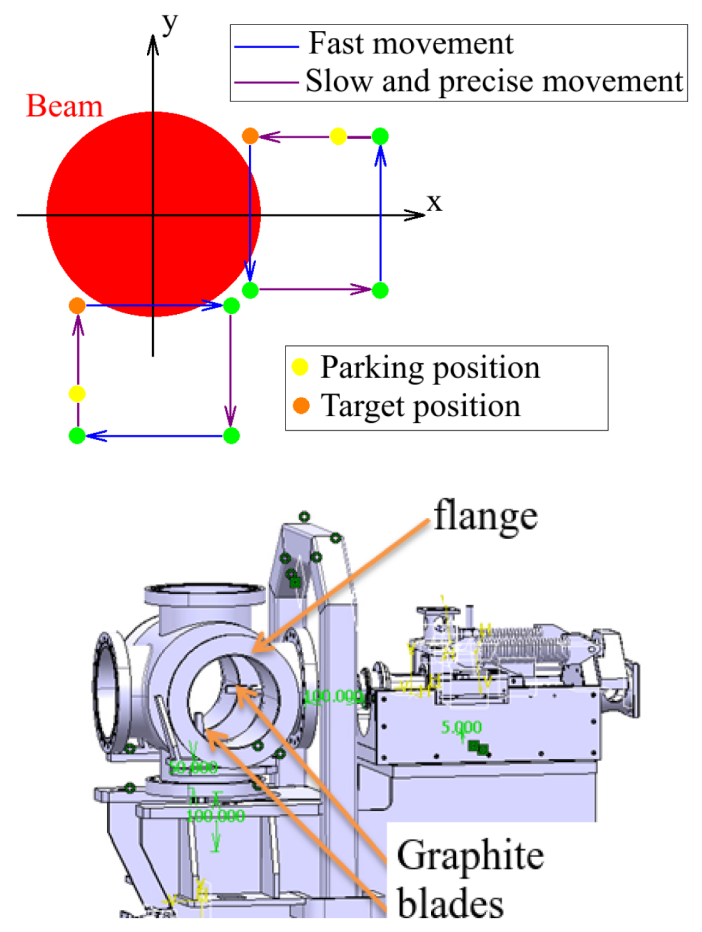

FIG. 3. Upper frame: schematics of the mechanical cycle of the blades of the SPS scrapers. Lower frame: 3D view of an SPS scraper tank and its mechanics. 
can be set to intercept the entire beam core; in order not to induce damage to the blades, low-intensity beams are used, e.g., with up to some $10^{12}$ protons. An example of past scraper scans can be found in Ref. [16].

\section{B. Monitors}

Readouts from SPS monitors [9] were collected during the setting up of the test; in this study, they are used for the proper setting up of the simulations and the benchmarking of the simulation tool. In particular, signals from the beam current transformer (BCT) were used to reconstruct the actual conditions of scraping during the test, like beam distribution, speed, and possible tilt of the blades; BLM signals around the ring were used as observable for actually benchmarking simulations.

The BCT records the beam intensity as a function of time. The hardware is based on a dc transformer. An analog-to-digital converter (ADC) digitizes the signal every $10 \mathrm{~ms}$, for a total of 1924 acquisitions. The acquisition starts $625 \mathrm{~ms}$ after the beginning of the cycle (see Fig. 2). Table I summarizes the main parameters of the BCT signals.

The SPS BLMs are nitrogen-filled ionization chambers [17], aimed at detecting secondary particle showers following the local loss of a beam particle. They are distributed all around the ring, for a total of 268 monitors. Most of them are assigned to each half-cell of the accelerator; additional ones are installed in the LSSs, in number and positions which depend on the purpose of the LSS and hence the devices there installed.

Each BLM is equipped with readout electronics and an 11-bit ADC system. The calibration factor changes according to the location of the BLMs (see Table II) to adapt the readout electronics to values measured during regular operation; as a consequence, the maximum signal they can read changes accordingly (see Table II).

BLM data are integrated over the entire SPS cycle. As a consequence, the readout of each BLM is affected by all loss mechanisms taking place during the SPS cycle that lead to local losses at the BLM. Hence, BLM readouts

TABLE I. Main parameters of the BCT signals. The calibration factor is used as least significant bit (LSB), for the estimation of the quantization errors.

\begin{tabular}{lc}
\hline \hline Parameter & Value \\
\hline Sampling rate & $100 \mathrm{~Hz}(10 \mathrm{~ms})$ \\
Total number of acquisitions & 1924 \\
Total duration of signal & $19240 \mathrm{~ms}$ \\
Delay with respect to SPS timing & $625 \mathrm{~ms}$ \\
Calibration factor & ${ }^{\mathrm{a}}$ \\
Analog bandwidth & $1.59 \times 10^{10}$ \\
\hline \hline a It should be noted that the calibration factors were derived by \\
parsing many BCT readouts and looking for the smallest change \\
in the signal, in absolute value.
\end{tabular}

TABLE II. Calibration factors ${ }^{\mathrm{a}}$ of the SPS BLMs and maximum signal they can read. The calibration factors are used as LSBs. There are no BLMs in LSS3.

\begin{tabular}{lcr}
\hline \hline Location & Calibration factors $\left[10^{-4} \mathrm{~Gy}\right]$ & \multicolumn{1}{c}{ Max [Gy] } \\
\hline Sextant 1/LSS1 & $1.140 / 12.7$ & $0.2335 / 2.6010$ \\
Sextant 2/LSS2 & $1.140 / 5.28$ & $0.2335 / 1.0813$ \\
Sextant 3/LSS3 & $1.104 / \cdots$ & $0.2261 / \cdots$ \\
Sextant 4/LSS4 & $1.100 / 1.14$ & $0.2253 / 0.2335$ \\
Sextant 5/LSS5 & $1.140 / 12.7$ & $0.2335 / 2.6010$ \\
Sextant 6/LSS6 & $1.100 / 5.28$ & $0.2253 / 1.0813$ \\
\hline \hline
\end{tabular}

${ }^{\mathrm{a}}$ As for the BCT, the calibration factors of the BLMs were derived by parsing many readouts and looking for the smallest change in the signal, in absolute value.

require processing in order to extract the signal actually due to scraping.

\section{THE ENDURANCE TEST}

The test of the SPS scraper blades was carried out to prove their endurance with beam. Therefore, the test envisaged to scrape the whole beam at full intensity to induce as high energy deposition as possible and hence magnify effects of damage. A microstructural analysis was done after dismounting the blades to reveal signs of damage.

\section{A. Test setup}

The test took place just before the start of the Long Shutdown 1 (LS1), on February 16, 2013, between 7:00 a.m. and 9:00 a.m. (local time at CERN, Geneva, Switzerland). The blades of the spare scrapers were tested, i.e., the BSHV.11759 (see Sec. II A); since they had been rarely used, disentangling possible signs of damage induced by the test from those induced by regular operation would be easier than with the operational blades.

The test foresaw to scrape the whole beam, i.e., at its center $(0 \sigma)$, at full intensity, i.e., at about $3 \times 10^{13}$ protons, to maximize the energy deposition in the blades. Both the horizontal and the vertical blades were tested, separately. The settings chosen for the test represent more an accident scenario rather than an operational one, as in the case of beam injection into the LHC only tails are scraped off; similarly, in case the scrapers are used to characterize the beam profile, diagnostics are not usually performed at full beam intensity (see Sec. II A).

Prior to the test, scraper scans (see Sec. II A) were taken on both planes to find the closed orbit at the scraper. The first position of each blade reducing the beam intensity by $100 \%$ was taken as that corresponding to the beam center (see Table III for both blades). The scans were carried out with low-intensity beams (about $10^{12}$ protons), to minimize the risk of damaging the blades prior to the test.

Immediately before and after each blade was tested, regular scraping (i.e., at $\sim 3.5 \sigma$, see Sec. II A) was 
TABLE III. Target positions used during the test $\left(z_{\text {test }}\right)$ and for regular scraping $\left(z_{\text {regular }}\right)$. The target positions are different by $2.2 \mathrm{~mm}$ on each plane.

\begin{tabular}{lcc}
\hline \hline Plane & $z_{\text {test }}[\mathrm{mm}]$ & $z_{\text {regular }}[\mathrm{mm}]$ \\
\hline Horizontal & -11 & -13.2 \\
Vertical & 5.7 & 3.5 \\
\hline \hline
\end{tabular}

performed with full intensity beams, to detect any change in performance. In addition to the target position of the test, Table III also reports those for regular tail scraping.

Since the scraper scans were primarily meant to determine the position of the closed orbit and not to characterize the beam shape, neither are the number of scraping positions particularly abundant nor are they equally spaced. Nevertheless, data can be fitted to provide a description of the circulating beam, which will be used for the simulation benchmarking (see Sec. IVA 2).
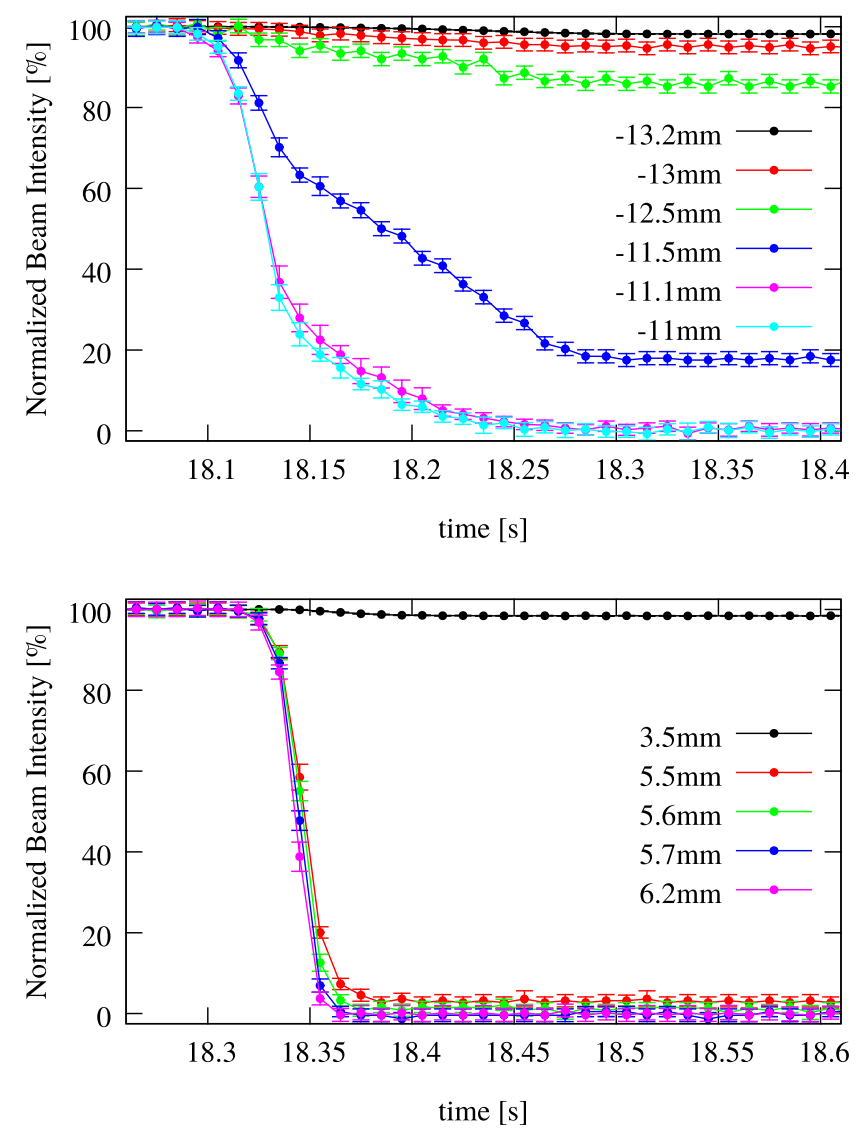

FIG. 4. Normalized BCT signals at low beam intensity obtained during the scraper scans with the horizontal (upper frame) and vertical (lower frame) blades. The shown curves are the average over consecutive fills at the same target position. When visible, error bars refer to the maximum between the dispersion of the sets of data used to compute the averages and the error propagation. It should be noted that the positions in the legend refer to the calibration of the blade mechanics, not to the closed orbit.
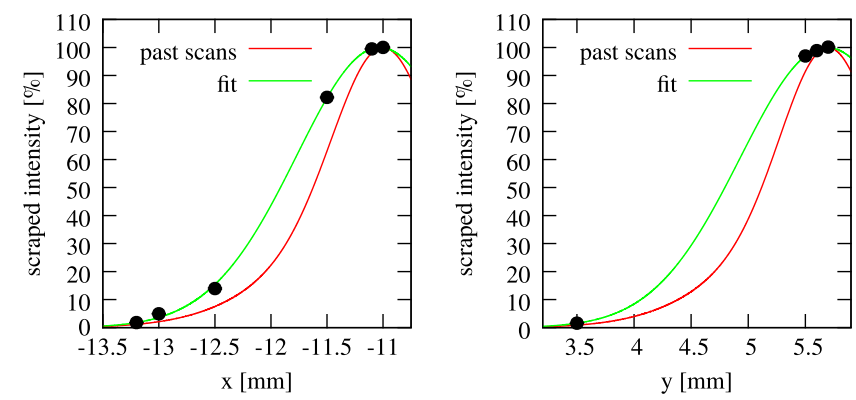

FIG. 5. Scraper scans performed before the test to determine the closed orbit at the scrapers: horizontal (left frame) and vertical (right frame) planes. The red curves are obtained from past scans [16] using emittances as measured by the WSs before the test and the centers as obtained by the scraper scans (see Table III). The green curves are the fits of Eq. (1) through the data; the fitting parameters are reported in Table IV.

Figure 4 shows the normalized BCT signals, i.e., relative to the beam intensity immediately before scraping, taken during the scraper scans. Each blade position during the scan was kept for few consecutive fills, to take into account fill-to-fill variations of the closed orbit. Figure 5 shows the results of the scraper scan. As expected, the beam had a bell-shaped distribution on both planes. Wire scans (WSs) were performed before the test to measure the normalized emittance of the beam core; they are reported in Table IV. Past measurements [16] have shown that the transverse distribution of the SPS beam at flattop follows a double Gaussian distribution that can be expressed as

TABLE IV. Normalized emittances as from the WS measurements and parameters of the curves shown in Fig. 5: "fit" refers to the fit of Eq. (1) through the data (green curves in the referred figure), whereas "measured" refers to double Gaussian distributions previously measured in SPS [16] and scaled with the emittance in machine (red curves in the referred figure). Errors of the fitting curves on the vertical plane are not reported since they are 0 , because the number of fitting parameters is equal to the number of data points. Values of betatron $\sigma$ at $450 \mathrm{GeV} / c$ as from linear optics and normalized emittances available in machine are shown for comparison.

\begin{tabular}{lccc}
\hline \hline & $\begin{array}{c}\text { Horizontal plane } \\
\text { Fit }\end{array}$ & $\begin{array}{c}\epsilon_{\mathrm{N}}[\mu \mathrm{m}] \\
\text { Measured }\end{array}$ & $\begin{array}{c}2.274 \\
\text { Optics }\end{array}$ \\
\hline$I_{1}[]$ & $0.0626 \pm 0.731$ & 0.632 & 1 \\
$\sigma_{1}[\mathrm{~mm}]$ & $0.518 \pm 1.79$ & 0.431 & 0.520 \\
$\sigma_{2}[\mathrm{~mm}]$ & $0.772 \pm 0.0832$ & 0.837 & $\cdots$ \\
$z_{0}[\mathrm{~mm}]$ & $-11.03 \pm 0.109$ & -11 & $\cdots$ \\
\hline
\end{tabular}

\begin{tabular}{lccc}
\hline \hline \multicolumn{1}{c}{ Vertical plane } & $\begin{array}{c}\epsilon_{\mathrm{N}}[\mu \mathrm{m}] \\
\text { Measured }\end{array}$ & $\begin{array}{c}1.874 \\
\text { Optics }\end{array}$ \\
& Fit & 0.63 & 1 \\
$I_{1}[]$ & 1 & 0.398 & 0.495 \\
$\sigma_{1}[\mathrm{~mm}]$ & 0.760 & 0.805 & $\cdots$ \\
$\sigma_{2}[\mathrm{~mm}]$ & $\ldots$ & 5.7 & $\cdots$ \\
$z_{0}[\mathrm{~mm}]$ & 5.69 & \\
\hline \hline
\end{tabular}




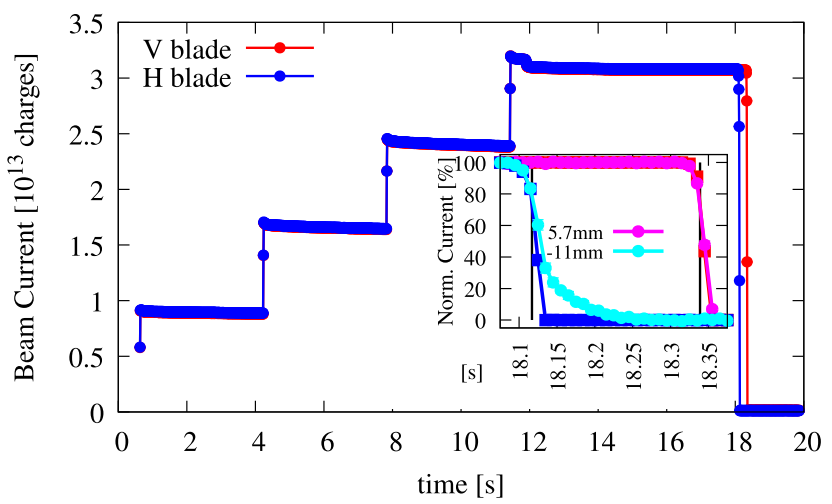

FIG. 6. BCT signals during the endurance test of the horizontal (blue curve) and the vertical (red curve) blades. The inset, focused on the moment of scraping, compares the normalized signals at scraping from the test (dark colors) to those obtained with lowintensity beams (light colors) for the same scraper settings (see Sec. III A and Fig. 4). When visible, error bars on the signal from the test refer to the LSB. The black vertical bars in the inset mark the time of the dump.

$$
\begin{aligned}
\operatorname{pdf}(z)= & \frac{I_{1}}{\sigma_{1}^{2}} \exp \left[-\frac{\left(z-z_{0}\right)^{2}}{2 \sigma_{1}^{2}}\right] \\
& +\frac{1-I_{1}}{\sigma_{2}^{2}} \exp \left[-\frac{\left(z-z_{0}\right)^{2}}{2 \sigma_{2}^{2}}\right],
\end{aligned}
$$

where $I_{1}$ is a relative weight, $z$ can be either $x$ or $y, z_{0}$ is the beam closed orbit at the scraper on the concerned plane, and the subscripts are used to identify the two single Gaussian distributions. Variances to the square appear at the denominator as a result of integrating in the $z-z^{\prime}$ phase space.

The green curves in Fig. 5 are the fits of Eq. (1) through the data; the fitting parameters are reported in Table IV. The red curves are obtained from past scans [16] using the emittances measured by the WS before the test and the centers obtained by the scraper scans (see Table III). The beam seems to be broader than expected, and with a distribution closer to a single Gaussian than to a double Gaussian, contrary to previous findings.

\section{B. Measurements taken during the test}

Figure 6 shows the BCT signal during the test of each blade. The inset, focused on the moment of scraping, compares the normalized signals from the test to those obtained with low-intensity beams for the same scraper settings, taken during the scraper scans.

The signals from the test are characterized by a sharp drop down at the moment of scraping, very similar to that of a beam dump. According to the CERN logging [18], the beam was dumped while sweeping the blades; therefore, testing of both blades ended prematurely. On the contrary, the profiles measured with low-intensity beams are not affected by any premature beam dump; hence, they show what should have been seen during the test. In the case of the horizontal blade, the two curves almost coincide before the dump, whereas they are quite distinct afterwards; as will be seen in Sec. IV A 3, the long tail of the BCT signal is due to the tilt of the blade. In the case of the vertical blade, the two curves are always in good agreement, with no evident difference after the dump trigger, hinting to an almost perfectly aligned blade. The total beam intensity right before scraping was $3.06 \times 10^{13}$ protons for both blades.

Figure 7 shows the time profile of the vacuum at the MKD dump kickers, located immediately upstream of the blades. Two distinct vacuum spikes are visible, synchronous with the movement of the blades during the test; the spikes cover some orders of magnitude in a very short time, indicating an abrupt emission of material, responsible for the degradation of the vacuum. These spikes are compatible with local sublimation of graphite induced by the high levels of energy density reached during the test.

Figure 8 shows the pattern of BLM signals during the test. The BLM at $\sim 560 \mathrm{~m}$ is immediately downstream of the scrapers; its readouts are extremely close to the limit given in Table II, indicating some possible saturation taking place. Being the BLM with the highest readout and the

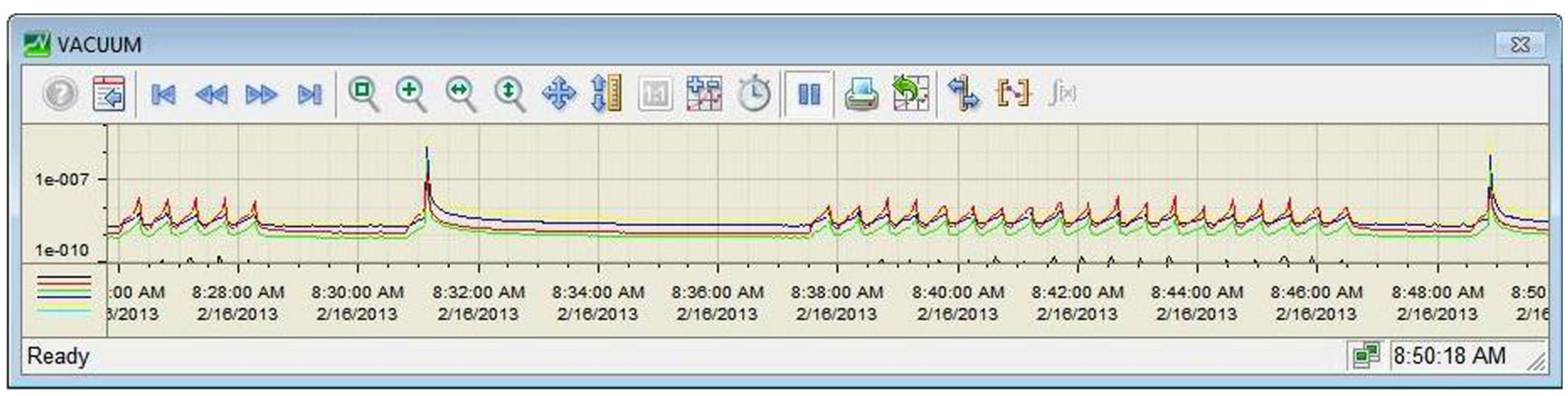

FIG. 7. Time profile of the vacuum at the MKD dump kickers immediately upstream of the blades under test. The vacuum spikes at 08:31 a.m. and 08:49 a.m. (local time at CERN, Geneva, Switzerland) are in conjunction to the test of the vertical and horizontal blades, respectively. All other small spikes happened at every scraping action when checking the performance of the blades with operational settings before and after the test. 
V blade $\quad$ H blade no scraping

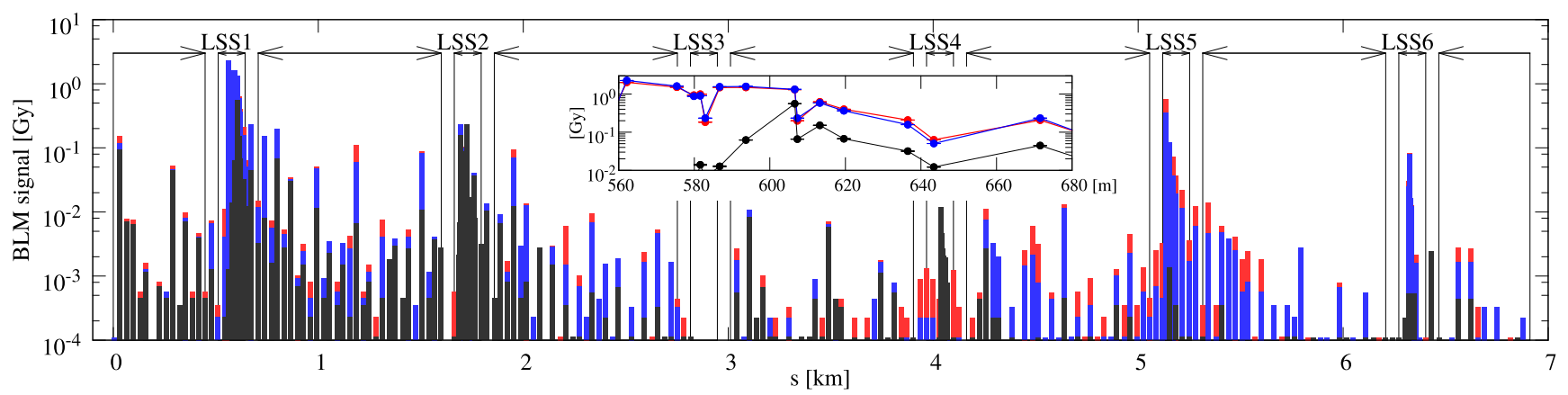

FIG. 8. BLM readouts along the SPS ring recorded during the endurance test of the vertical (red curve) and of the horizontal (blue curve) blades. For comparison, a pattern recorded with beam at full intensity but no scraping is shown as well (black curve) for a dump. The inset focuses on the LSS1 downstream of the BSHV.11759 scrapers under test, located at $s=\sim 560 \mathrm{~m}$.

closest to the scrapers, it is almost sure that during both tests it triggered the premature beam dump. The two BLMs at $\sim 580$ and $\sim 610 \mathrm{~m}$ belong to those regularly distributed around the ring; their readout is also very close to the limit reported in Table II. The three BLMs in between seem to be saturating as well, with an almost flat pattern. For comparison, a BLM pattern obtained at full beam intensity for a dump with no scraping is shown in the same figure. The pattern is characterized by a marked peak at the dump, given by the closest BLM, located at $\sim 600 \mathrm{~m}$, and few other downstream BLMs. The shift between the BLM patterns from the test and that with no scraping shows the importance of the direct contribution from scraping over that of the dump.

Figure 9 shows the normalized BCT signals during regular scraping before and after the endurance test of the blades. The figure shows a negligible worsening of the scraping performance of the blades following the test.

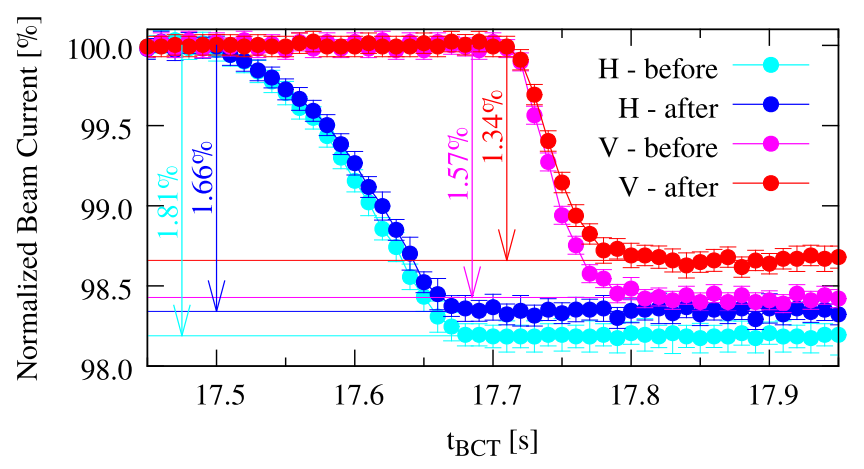

FIG. 9. Normalized BCT signals during regular scraping before (light colors) and after (dark colors) the test of the horizontal (blueish curves) and vertical (reddish curves) blades. Each curve was obtained as an average of a few consecutive fills. The error bars refer to the maximum between the dispersion of data and error propagation.

\section{Microstructural analysis of the tested blades}

After the test, the blades were dismounted and underwent a microstructural analysis to detect signs of damage. At removal, the overall shape of the blades was untouched, but signs of change of crystallographic state were visible around the edge actually facing the beam (see Fig. 10, left frame).

The SEM analyses [19] identified a change in the material porosity, largest at the edge of the blade (see Fig. 10, right frame, region 1, on the left) and gradually approaching the value of $2 \%$ of a region not affected by the beam, taken as reference. In particular, three regions with different porosity levels could be identified (see Table V). The estimation of the porosity was performed with a visual technique, based on the difference in contrast visible in the SEM picture between the bulk material (light color) and the porous one (dark color).

It should be noted that a decrease in the density, corresponding to an increase in porosity, translates into an increase of the inelastic interaction length of beam protons, which may lead to a degradation of performance of the blade.
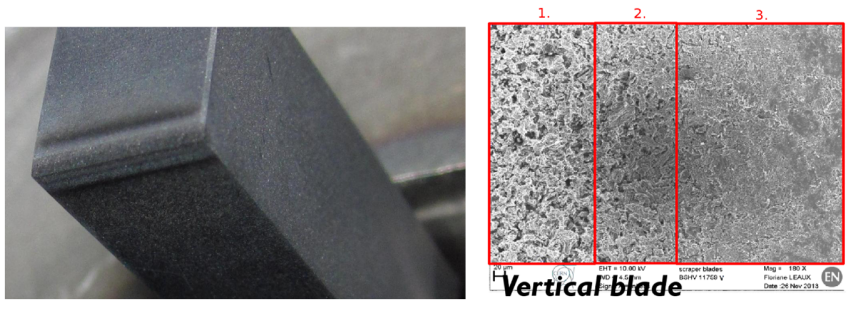

FIG. 10. Vertical blade of the tested scraper as when dismounted (left frame) and as visualized with the SEM [19] (right frame). The SEM image is zoomed on the region around the chamfer in view of the beam and hence affected by the burst. Three zones with different levels of porosity were identified. The horizontal blade shows a very similar degradation of the porosity. 
TABLE V. Estimated porosity of the tested blades following the endurance test [19] as measured in the three regions shown in Fig. 10, right frame.

\begin{tabular}{lccc}
\hline \hline & \multicolumn{3}{c}{ Porosity [\%] } \\
\cline { 2 - 4 } Blade & Region 1 & Region 2 & Region 3 \\
\hline Horizontal blade & 28 & 7 & 9 \\
Vertical blade & 31 & 10 & 9 \\
\hline \hline
\end{tabular}

\section{Estimation of amount of scraped beam}

In order to reconstruct the levels of energy deposition reached during the test, it is essential to estimate the amount of beam actually scraped, since the beams were prematurely dumped (see Sec. III B). In the following, two methods are presented: one is based on the BCT signals, whereas the other one on the BLM measurements.

The estimation based on the BCT signals is obtained interpolating the normalized signals from the test at the times of dumping (see Fig. 6). With this method, it is found that $20 \%$ and $30 \%$ of the beam intensity was scraped by the horizontal and vertical blades, respectively, corresponding to $6.1 \times 10^{12}$ and $9.2 \times 10^{12}$ protons, respectively. These estimations are affected by the limited analog bandwidth of the BCT and the frequency of logging, which are not optimized for measuring the beam intensity with accuracy in case of fast events like scraping; nevertheless, these estimations show that a relevant fraction of the initial-beam intensity was actually dumped and did not contribute to generating the damage.

The estimation based on the BLM measurements requires additional processing. In fact, BLM signals are integrated over the entire SPS cycle, and since the SPS LSS1 hosts the scraping, injection and dumping systems, any LSS1 BLM can integrate the signal coming from beam injection, scraping, and dumping. Therefore, it is important to properly disentangle the BLM signal due to scraping from that due to beam injection or dumping. Since the beams were cleanly injected into the SPS and the beam was lost either via scraping or via beam dumping, injection losses can be neglected.

Since the SPS BLMs are ionization chambers, readouts scale linearly with the number of protons locally lost, provided that readouts are far from saturation. Moreover, each mechanism of loss yields to a particular BLM pattern, which can be qualitatively different from the others. Hence, clean and nonsaturated signals obtained from fills where either the beam was fully scraped with no dump or those obtained in the exactly opposite condition could be linearly combined to reconstruct patterns obtained during cycles where the beam was dumped after scraping, as during the test. The signal $R_{i}$ read by the $i$ th BLM (e.g., in one of the test cycles) where a portion $s$ of the beam is scraped and the rest is dumped can be estimated as

$$
R_{i}=s R_{i, s}+(1-s) R_{i, d} .
$$

$R_{i, s}$ and $R_{i, d}$ are the signals in that same BLM for full beam scraping and in case of dump, respectively. It should be noted that $R$ signals are normalized to the beam intensity. It is important to underline that, unlike BCT readouts, BLM signals are loosely sensitive to details on the scraping conditions, like beam distribution and orbit jittering, $50 \mathrm{~Hz}$ jitter due to the power supply network, blade position, speed and tilt angle, etc. If the three measurements for a specific BLM are available, it is possible to deduce the amount of beam scraped. Other studies [20] have been carried out deploying machine learning techniques, which find increasing applications in particle accelerators at the intensity frontier, as proven by a recent high level whitepaper [21].

Figure 11 shows the pattern of BLM signals obtained for full beam scraping with full beam intensity (i.e., during the test) and with low-intensity beams, zoomed on LSS1. Signals with no scraping for the same beam intensities are also shown. The BLM signals for full beam scraping with low-intensity beams (lower frame in Fig. 11) and those for full beam dumping with high-intensity beams (upper frame in Fig. 11) can be used in Eq. (2), since they are far from saturation. Similarly, BLM signals far from saturation can be used to deduce the amount of beam scraped during the test. Since most of the BLMs during the test were at saturation (see Sec. III B), only BLM.11836 can be taken among the BLMs largely sensitive to scraping, and BLM.11954.MKP4 and BLM.120 among the BLMs largely sensitive to dumping. These BLMs are the only ones with their signals above the noise level or not saturating in all the concerned configurations, i.e., testing, scraping with low-intensity beams, and dumping with lowand high-intensity beams.

Figure 12 shows the pattern of the BLM signals measured during the endurance test of each blade, and those reconstructed with Eq. (2); the latter correspond to the signals that should have been seen during the test if no premature beam dump occurred and the dynamic range of the electronics was larger. Matching the aforementioned BLMs yields to $41.5 \%$ and $45 \%$ of the beam intensity being scraped by the horizontal and vertical blades, respectively, corresponding to $1.27 \times 10^{13}$ and $1.7 \times 10^{13}$ protons, respectively.

Given the good properties of linear scaling of BLM signals on total number of scraped protons and their limited sensitivity on details of the process of scraping (e.g., distribution of the beam actually in the machine, settings of the blade like speed and tilt angle, etc.), the estimations of the amount of beam scraped during the test before the dump seem to be quite robust. They are approximately $80 \%$ and $50 \%$ larger than those obtained with the BCT signals for the horizontal and vertical blade, respectively, with a corresponding increase in the estimated 

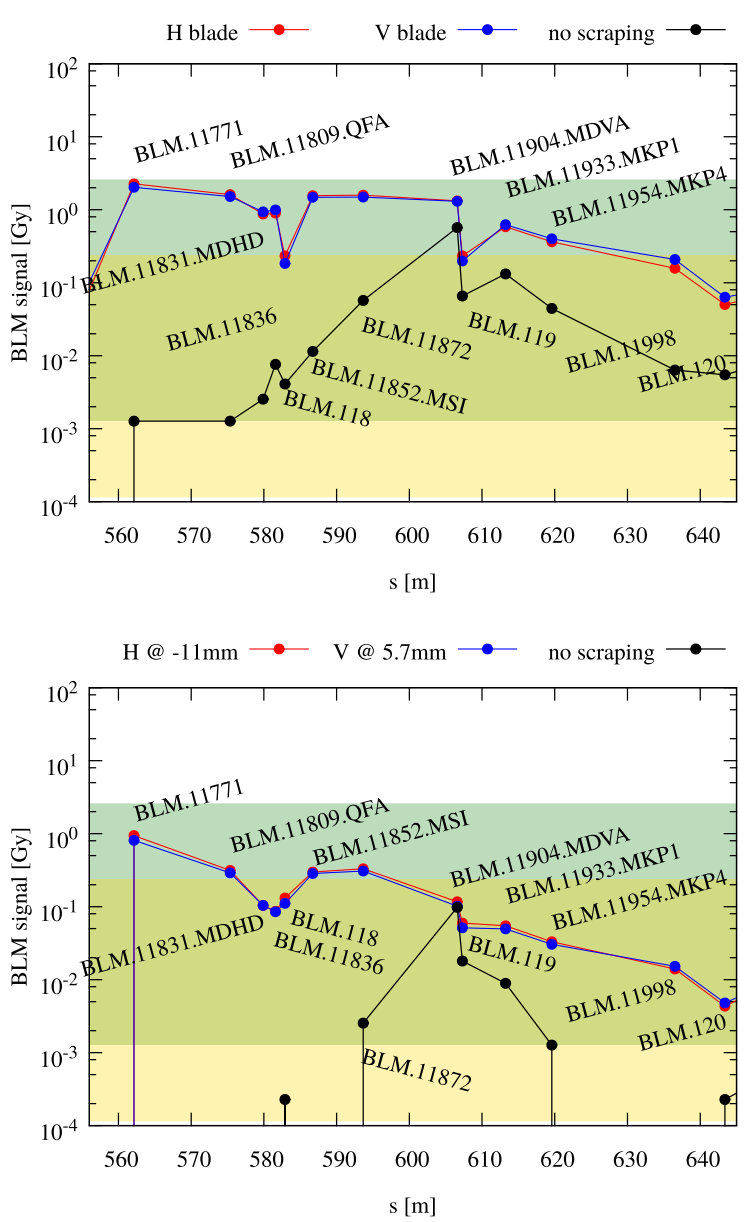

FIG. 11. Pattern of BLM signals in LSS1 obtained with the blades for full beam scraping during the endurance test (upper frame) and with low-intensity beams (lower frame). Signals for full beam dumping with no scraping at all at the same intensity are shown as well. The range of signal for regular cell BLMs and for the LSS1 BLMs is shown by the yellow and blueish bands, respectively.

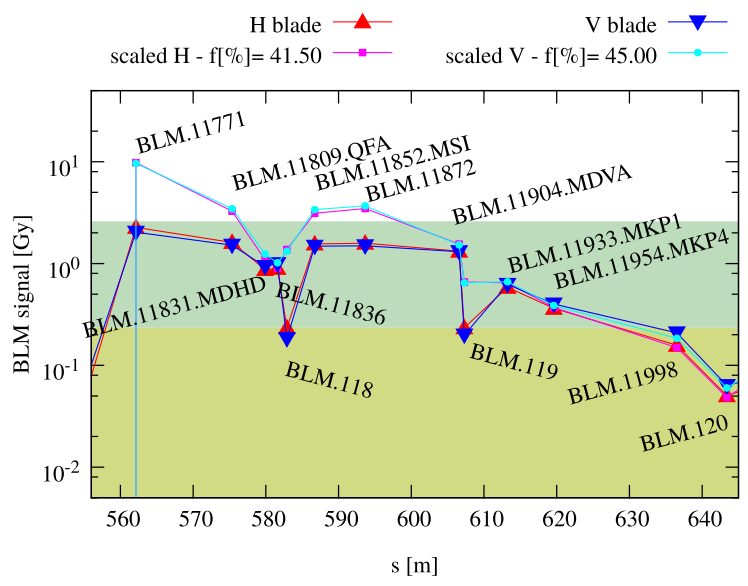

FIG. 12. Pattern of BLM signals in LSS1 during the endurance test of each blade, as measured (dark-colored curves) and as reconstructed with Eq. (2) (light-colored curves): horizontal (reddish curves) and vertical (blueish curves) blades. The percentage in the key reports the reconstructed amount of scraped beam.
TABLE VI. Summary of amount of beam scraped as estimated via the $\mathrm{BCT}$ and BLM signals.

\begin{tabular}{lcc}
\hline \hline & \multicolumn{2}{c}{ Intensity } \\
\cline { 2 - 3 } Blade & BCT/BLM [\%] & BCT/BLM $\left[10^{13}\right]$ \\
\hline Horizontal & $20 / 41.5$ & $0.61 / 1.27$ \\
Vertical & $30 / 45.0$ & $0.92 / 1.37$ \\
\hline \hline
\end{tabular}

energy deposition. Table VI summarizes the results with both methods.

\section{E. Main outcomes}

Even if the endurance test was prematurely terminated by the BLMs while scraping, signs of damage have been found in the scraper blades thanks to the SEM analyses. During the test, intense vacuum spikes of some orders of magnitude were seen when the blades were tested, compatible with an abrupt sublimation of material from the blades, in accordance with the SEM analyses. Nevertheless, even if the blades were damaged, a negligible change in scraping performance of the blades was observed following the test. The amount of beam scraped before the dump was estimated with two methods, which both showed that only some tens of $\%$ of the beam was scraped and the rest was dumped. The amount of beam actually scraped during the test will be used to reconstruct in simulations the levels of energy deposition actually reached in the blades.

\section{SIMULATIONS AND BENCHMARK}

Simulations were mainly needed to estimate the levels of energy deposition reached during the test and hence have a correlation to the induced damage. Such a benchmark is important to address operational limits within which the scrapers can be safely operated or damage can be tolerated.

Given the short length of the blades with respect to the inelastic scattering length (see Table VII), beam protons at flattop $(450 \mathrm{GeV} / c)$ need many turns on average before undergoing a nuclear scattering event leading to their loss-i.e., about 45 . Therefore, it is essential to accurately describe the evolution of the impact position of protons turn by turn. To do so, the simulation setup must properly take into account the single particle beam dynamics in the accelerator and predict the scattering angles experienced by protons when going through the scrapers turn after turn

TABLE VII. Inelastic interaction length $\lambda_{I}$, radiation length $X_{0}$, and average ionization energy loss $-\mathrm{d} E / \mathrm{d} x$ of $450 \mathrm{GeV} / c$ protons in graphite used for the scraper blades. Values were computed by FLUKA.

\begin{tabular}{lcc}
\hline \hline$\lambda_{I}$ & $X_{0}$ & $-\mathrm{d} E / \mathrm{d} x$ \\
\hline $44.5 \mathrm{~cm}$ & $23.3 \mathrm{~cm}$ & $3.22 \mathrm{MeV} / \mathrm{cm}$ \\
\hline \hline
\end{tabular}


("multiturn" effects) before being definitively lost. For this reason, simulations were carried out with the FLUKAsixTrack coupling, which combines the refined physics models of the two codes for most accurate results. Moreover, the movement of the blades, responsible for the concentration of the energy deposition on the edge in direct view of the beam, was taken into account changing the blade position in the FLUKA geometry turn by turn.

In a FLUKA-SixTrack coupled simulation, the beam is tracked turn by turn through the accelerator lattice by sixTrack; at the location of interest, the tracked particles are transferred to FLUKA and transported through its 3D geometry; beam particles surviving the interaction with matter are sent back to sixTrack to continue their tracking through the accelerator lattice and hence estimate the distribution of losses around the ring. Particles are exchanged among the two codes by means of Flukaio, a $\mathrm{C}++$ library implementing the TCP/IP communication protocol.

The endurance test offered the opportunity for an extensive benchmark of the simulation tool. The benchmark is carried out as follows. (1) The time evolution of the beam intensity predicted by the simulation is quantitatively compared to BCT signals. Those from the scraper scans (see Sec. III A) are used. Matching simulation results to the BCT signals by varying tilt angle and speed of the blades allowed to identify those occurring during the test, leading to a more accurate estimation of the energy deposition in the blades. (2) Losses of protons in the machine aperture along the entire ring predicted by simulations are qualitatively compared to BLM patterns around the ring. A quantitative comparison is done only for LSS1.

\section{A. Benchmark against BCT readouts}

\section{Simulation settings}

Beam tracking through the SPS lattice was carried out by sixTrack considering the Q20 optics at flattop energy, i.e., $450 \mathrm{GeV} / c$. The permanent orbit bump in LSS1, necessary to ease dumping [22], was taken into account. Losses around the ring were estimated checking the position of the tracked particles against the SPS aperture model during tracking ("online aperture checking").

The interaction of the beam with the scraper blades was carried out by FLUKA. The geometry mainly consisted of the scrapers, i.e., the BSHV.11759 and the BSHV.11771, each equipped with two blades, with realistic shape, dimensions, material density, and speed (see Sec. II A). Other beam-intercepting devices were taken into account, to improve the description of the SPS aperture and take into account possible reinteractions of beam protons already scattered by the scraper blades, but they are not described here for the sake of brevity. The energy deposition was estimated by means of two Cartesian meshes on each blade, i.e., a coarse one, with a transverse binning stepped by $50 \mu \mathrm{m}$ on the horizontal and vertical dimensions and by $500 \mu \mathrm{m}$ longitudinally, and a more refined one, with a transverse binning smaller by a factor 5 on the two transverse dimensions.

\section{Beam shape}

For the consistency of the benchmark, the beam distribution used in simulations was that obtained by fitting the scraper scans performed before the test (see Sec. III A); these are shown in Fig. 5.

Since no information about the beam characteristics on the longitudinal plane was available during the test, typical figures are assumed [9], i.e., the sampled beam is arbitrarily given a Gaussian distribution in momentum, with a root mean squared relative momentum spread $\sigma_{\delta}=10^{-4}$. The fact that the beam profile reconstructed with the scraper scans is very close to a Gaussian beam might raise the doubt that the reconstructed beam distribution reflects the beam distribution in momentum. This is not the case, since the value of dispersion at the scrapers is relatively small, and the momentum spread should be so large that only a small fraction of the population could fit into the bucket. This argument is even stronger if applied to the vertical plane, where the dispersion is practically 0 .

\section{Results}

Figure 13 compares the time evolution of the beam population during scraping as predicted by the simulation and as from BCT signals (see Fig. 4). For each blade, three scraping positions are shown: full beam scraping, i.e., at $0 \sigma$; regular scraping, i.e., with the blades off the beam center by $2.2 \mathrm{~mm}$ (see Table III); and scraping at an intermediate position. Three combinations of speed and tilt angles have been explored. Only positive tilt angles about the longitudinal axis are shown, since the negative ones are not able to reproduce the shape of the time evolution of the beam intensity for the horizontal blade at full beam scraping. For the speed, the nominal value (see Sec. II A) has been considered along with a lower one to give a hint of the sensitivity.

The tilt angle plays a key role for matching predictions from simulations to measurements for the horizontal blade. In particular, it is essential to explain the qualitative change of shape of the BCT signals for full and intermediate scraping positions after $\sim 17.5 \mathrm{~s}$, marking a change in scraping regime. In fact, at that point, the part of the blade actually performing the scraping moves from the lower edge intercepting tails to the innermost face intercepting the core (see schematics in Fig. 14). The second regime is slow, since it needs the whole blade face to reach the beam center before cleaning is accomplished; moreover, it is not fully efficient, since protons the closest to the core see the blade only by the tiny, innermost edge 

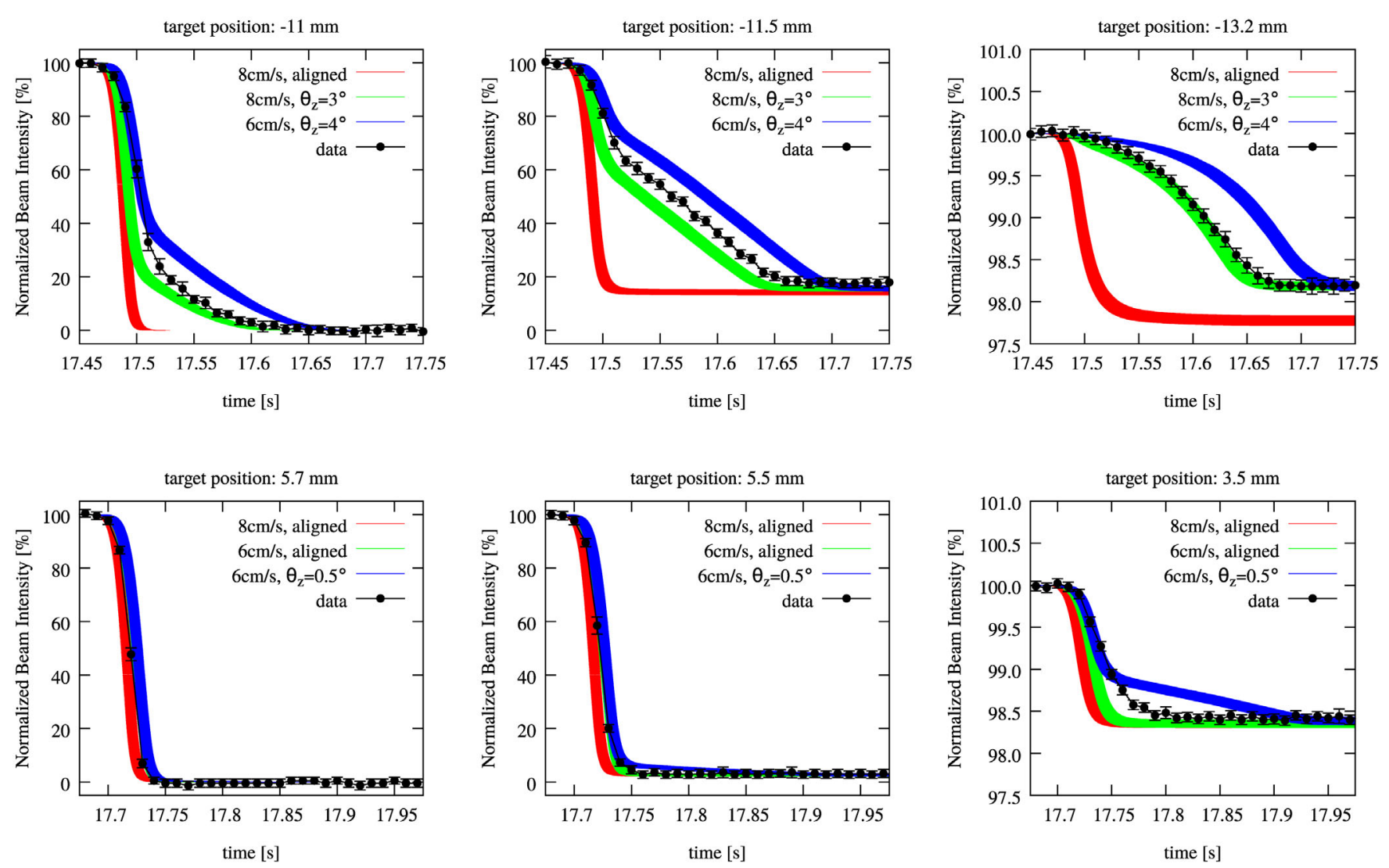

FIG. 13. Comparison of the time evolution of the beam intensity during scraping as predicted by the simulation and as from BCT signals (see Fig. 4): results for the horizontal (top frames) and the vertical (lower frames) blades. For each blade, three scraping positions are shown: full beam scraping (left frames), regular scraping (right frames), and scraping at an intermediate position (central frames). Three combinations of blade speed and tilt are shown. The errors on the simulation results refer to the statistical error only.

(the magenta one in the figure) for very few turns, with a total path length in the blade substantially smaller than the inelastic scattering length, and hence not sufficient to lead

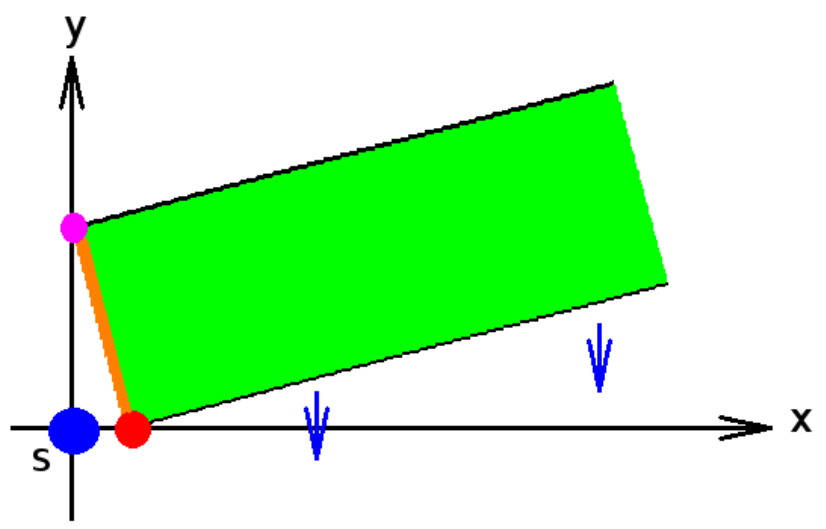

FIG. 14. Schematics of the horizontal scraper blade tilted by a (positive) angle about the longitudinal axis. The blue arrows show the movement of the blade. The beam comes out of the page at the blue point. The red point marks the lowest edge of the blade (parallel to the beam), the magenta point marks the innermost edge (parallel to the beam), and the orange line marks the innermost face. to actual losses. In fact, the tilt angle is responsible for the different amount of surviving beam for regular scraping. As expected, speed mainly changes the duration of the process.

The BCT patterns taken with the vertical blade are qualitatively different from those taken with the horizontal one, being more dependent on speed rather than on angle. The simulated pattern with the angle is shown more for the sake of completeness rather than for the need to explain a pattern in the measurements.

Table VIII summarizes the values of speed and tilt angles of the tested blades as reconstructed by comparing simulations to measurements (see Fig. 13). The explored values catch a realistic interval of tilt angle and speed of the

TABLE VIII. Speed and tilt angle of the tested scraper blades as reconstructed from the benchmark of simulation results against the BCT measurements.

\begin{tabular}{lcc}
\hline \hline Blade & Speed $\left[\mathrm{mm} \mathrm{s}^{-1}\right]$ & Tilt angle $\left[{ }^{\circ}\right]$ \\
\hline Horizontal & $60-80$ & $3-4$ \\
Vertical & $\leq 60$ & $<0.5$ \\
\hline \hline
\end{tabular}


horizontal blade. The vertical blade seems to be properly aligned, but the speed appears to be substantially smaller than the nominal one.

It should be noted that the range of angles identified for the horizontal blade is larger than expectations from the design of the mechanics [23], even when the contributions from all possible sources of tilt are summed up in the most pessimistic and unrealistic assumptions. This discrepancy may be the result of a wrong alignment of the whole mechanics. Another explanation could be that the joint which keeps in position the arm holding the horizontal blade may have been subject to wear; in fact, the arm, in stainless steel, is kept horizontal only by the joint to the mechanics generating the movement, and the joint may have suffered from the torque originated by the weight and length of the arm. Similarly, the fact that the vertical blade seems to be correctly aligned is compatible with the torque on the joint of the arm being extremely small, thanks to the vertical position of the arm.

\section{Estimation of energy deposition}

Table IX summarizes the energy deposition values estimated with the simulations at full beam scraping (see Sec. IV A), taking into account the amount of scraped beam as estimated via the BCT and BLM signals (see Table VI). The intervals of energy deposition consider all the combinations of tilt angle and speed, shown in the frames of Fig. 13. Values from the two scoring meshes deployed (see Sec. IV A 1) are reported for comparison; values obtained with the more refined mesh cover a wider range due to statistical fluctuations.

All the values are clearly above the sublimation heat for graphite, here assumed as $12.8 \mathrm{~kJ} \mathrm{~cm}^{-3}$, confirming that the blades were locally set in sublimation, generating the vacuum spikes recorded during the test. The sublimation heat of graphite is calculated as the heat necessary to reach the sublimation temperature $\left(T_{\text {high }}=3600^{\circ} \mathrm{C}\right)$ starting from room temperature $\left(T_{\text {low }}=22^{\circ} \mathrm{C}\right)$, integrating the curve of the specific heat of the considered graphite $c(T)$, shown in Fig. 15, as

TABLE IX. Summary of maximum energy deposition values expected in the blades during the endurance test. Values take into account the amount of scraped beam as estimated via the BCT and BLM signals (see Table VI). The quoted meshes have a longitudinal stepping of $500 \mu \mathrm{m}$.

\begin{tabular}{|c|c|c|c|}
\hline \multirow[b]{2}{*}{ Blade } & \multirow{2}{*}{$\begin{array}{l}\text { Mesh } \\
{[\mu \mathrm{m}]}\end{array}$} & \multicolumn{2}{|c|}{ Max } \\
\hline & & {$\left[\mathrm{GeV} \mathrm{cm}{ }^{-3}\right.$ per $\left.\mathrm{p}^{+}\right]$} & BCT/BLM $\left[\mathrm{kJ} \mathrm{cm}^{-3}\right]$ \\
\hline \multirow[t]{2}{*}{ Horizontal } & $50 \times 50$ & $14-16$ & $14-16 / 28-33$ \\
\hline & $10 \times 10$ & $20-25$ & $20-2$ \\
\hline \multirow[t]{2}{*}{ Vertical } & $50 \times 50$ & $13-16$ & $19-24 / 28-35$ \\
\hline & $10 \times 10$ & $18-25$ & $27-37 / 39-55$ \\
\hline
\end{tabular}

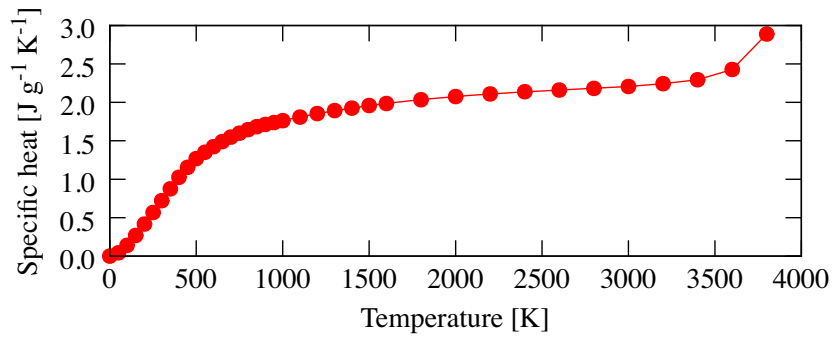

FIG. 15. Specific heat at constant pressure of graphite Steinemann R4550 as a function of temperature.

$$
\Delta E=\int_{T_{\text {low }}}^{T_{\text {high }}} c(T) \mathrm{d} T,
$$

where $\Delta E$ is the energy to be calculated. No latent heat is taken into account.

\section{B. Benchmark against BLM signals}

The benchmark against BLM signals is focused on full beam scraping only, since in this scenario the contribution to BLM signals from scraping is maximized.

A qualitative comparison between the BLM signals along the SPS ring and the losses predicted by simulations is given first. This comparison can only be qualitative, since the result of the simulation is a loss map, i.e., a histogram of proton losses along the ring, whereas the BLM signals are readouts of ionization chambers in presence of secondary particle showers due to protons hitting locally the machine aperture.

The focus is then moved to the LSS1, where the tested scrapers are located, for a quantitative comparison between the BLM readouts and energy deposition values in the BLMs predicted by simulations. This comparison can be quantitative as it relies on the simulation of the entire particle cascades taking place during scraping; consequently, all the mechanisms leading to energy deposition in the BLMs are taken into account. Being quantitative, this comparison is the most significant part of the benchmark of the FLUKA-SixTrack coupling.

\section{Qualitative comparison against BLM signals}

Figure 16 shows the qualitative comparison between the readouts from all the BLMs along the SPS during the endurance test of the horizontal blade and the corresponding beam loss map from simulations. The readouts obtained during the test of the vertical blade are not shown, as they are very similar (see Fig. 8). For comparison, the BLM readouts obtained with low-intensity beams are shown as well. Even though the beam intensity is lower by a factor $\sim 24$ with respect to that of the test, this additional case gives a more essential overview of the loss locations, since only the relevant BLMs stand up. The BLM readouts have been cleaned of the signal from other sources, subtracting from the original values the measurements without scraping 

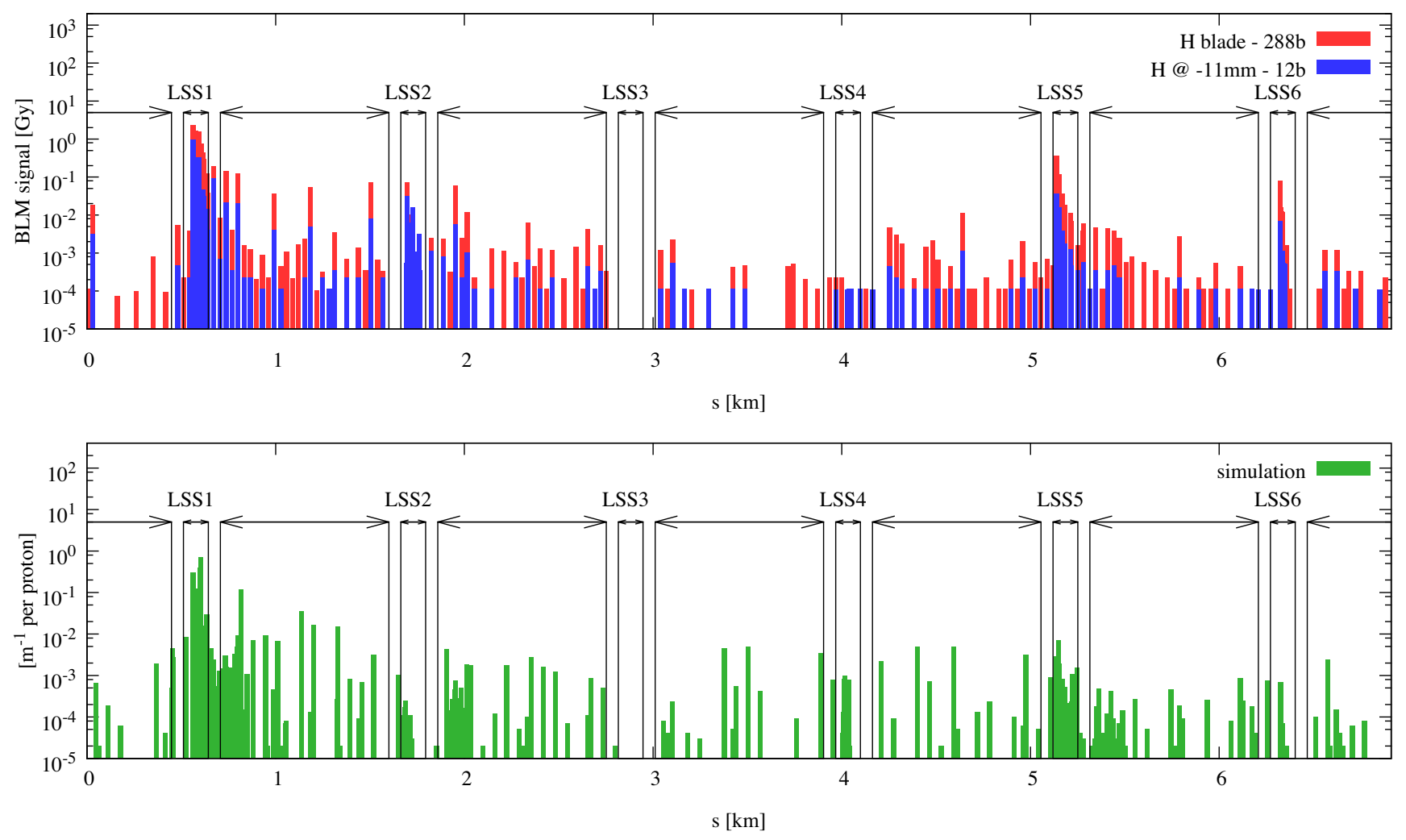

FIG. 16. Qualitative comparison between the readouts from all the BLMs along the SPS during the endurance test of the horizontal blade (upper frame, red curve) and the corresponding beam loss map from simulations (lower frame). For comparison, the BLM readouts obtained with low-intensity beams for the same scraping position are shown as well (upper frame, blue curve). BLM readouts have been cleaned of the signal from other sources, subtracting from the original values the measurements without scraping (see Fig. 8). No error bar is shown for the sake of clarity.

(see Fig. 8). As a consequence, the shown BLM patterns should represent the net effect due to scraping.

The loss map reproduces the main characteristics of the BLM pattern, i.e., only selected LSSs are most loaded, and arcs are affected by moderate losses. In particular, losses concentrate mostly in LSS1, where the scrapers are located, and LSS5. Losses in LSS5 and LSS6 are reproduced thanks to the explicit modeling in the FLUKA geometry of beamintercepting devices there installed (see Sec. IV A 1); while the machine aperture in LSS5 is quite wide with no obvious ways to improve the model, the aperture profile in LSS6 may be improved. Losses in LSS2 and LSS4 are moderate, as the readout of the BLMs there installed is. LSS3 is completely empty [24]. Losses in the arcs reproduce fairly
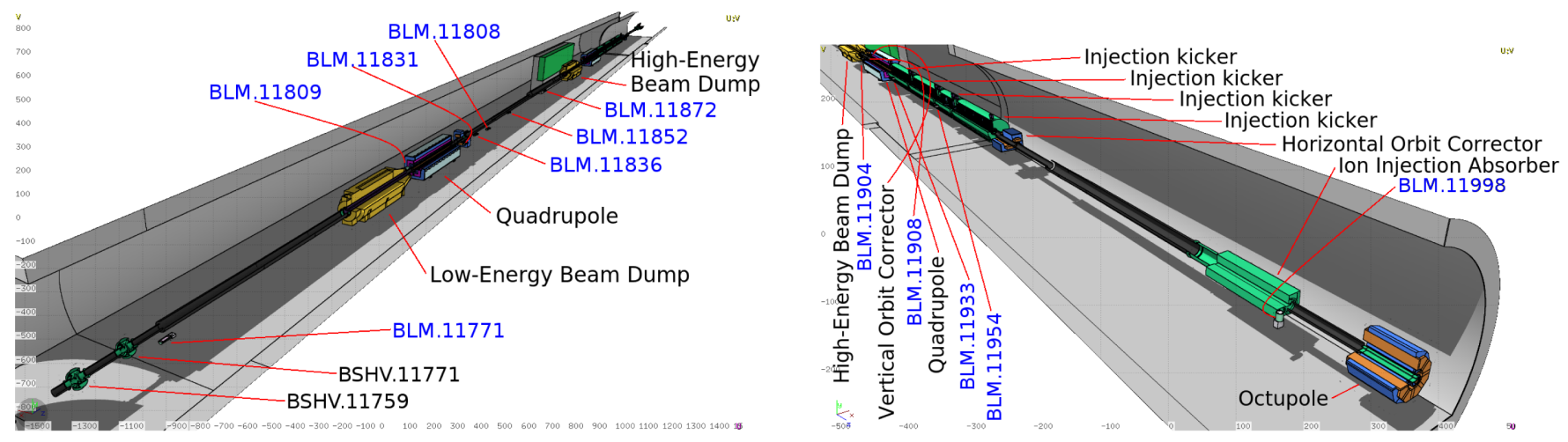

FIG. 17. 3D rendering obtained with the FLAIR geoviewer of the FLUKA geometry of LSS1: view from upstream (left frame) and from downstream (right frame). The beam travels from left to right. The scrapers are visible at the upstream edge of the upper frame. BLMs, despite not plainly visible in the geometry, are located usually at beam height. They are indicated as well, labeled in blue. 
TABLE X. Characteristics of the BLM active gas region as modeled in the FLUKA geometry.

\begin{tabular}{lccc}
\hline \hline Gas & Volume $\left[\mathrm{cm}^{-3}\right]$ & Density $\left[\mathrm{g} \mathrm{cm}^{-3}\right]$ & Mass $[\mathrm{g}]$ \\
\hline $\mathrm{N}_{2}$ & 1141.1 & 0.00126 & 1.4378 \\
\hline \hline
\end{tabular}

well the BLM pattern. The beginning of the arcs between LSS1 and LSS2, between LSS2 and LSS3, and between LSS5 and LSS6 see a local intensification of losses, as it also happens for the BLM signals. Local loss spikes in the arc take place nearby a spike in the BLM pattern, even though with a large variety in the ratio between the BLM readout and the intensity of the predicted loss. Therefore, the agreement between simulation results and measurements can be regarded as satisfactory.

\section{Quantitative comparisong against BLM signals in LSS1}

BLMs are sensitive to secondary particle showers, and all the details possibly affecting the development of the showers impact the estimation of the signals. Hence, an accurate model of the LSS1 downstream of the tested scrapers was implemented in the FLUKA geometry, covering a couple of lattice cells downstream of the scrapers, including magnets and their fields. Figure 17 shows a $3 \mathrm{D}$ rendering obtained with the FLAIR geoviewer [25] of the FLUKA geometry of the LSS1.

The accuracy of the estimated BLM signals strongly depends on monitor positions in the simulated geometry.
BLM positions were taken from the technical drawings of the SPS [26] and the lattice structure of the accelerator [9]. In some occasions, technical drawings are not consistent with each other; moreover, the uncertainty on the transverse positions can be even larger than the BLM dimensions. Since the endurance test was carried out at the end of the SPS activity in 2013 and afterwards the LSS1 was dismounted for important maintenance works, there is no way to resolve the uncertainty due to BLM positioning. Therefore, BLMs have been simulated in their nominal positions, i.e., as indicated by the most accurate drawings.

BLM signals are estimated as dose in the region of active gas inside the monitor. Table $\mathrm{X}$ summarizes the characteristics of the BLM gas region as modeled in the FLUKA geometry.

Apart from the more refined geometry of LSS1 and more relaxed transport thresholds, the same simulation settings as those presented in Sec. IVA 1 were used.

Figure 18 compares the BLM signals in LSS1 downstream of the scrapers and the predictions by simulations. BLM readouts obtained at full beam scraping with lowintensity beams (see Fig. 11) are used, since for lowintensity beams BLMs proved to perform in the regime of linear response (see Sec. III B). Predictions from simulations are shown in red, and take into account the permanent orbit bump in LSS1, whereas measurements are shown by the black curve. The agreement is remarkably good for some BLMs, whereas it is poorer for others, even though within a factor of $2-3$.
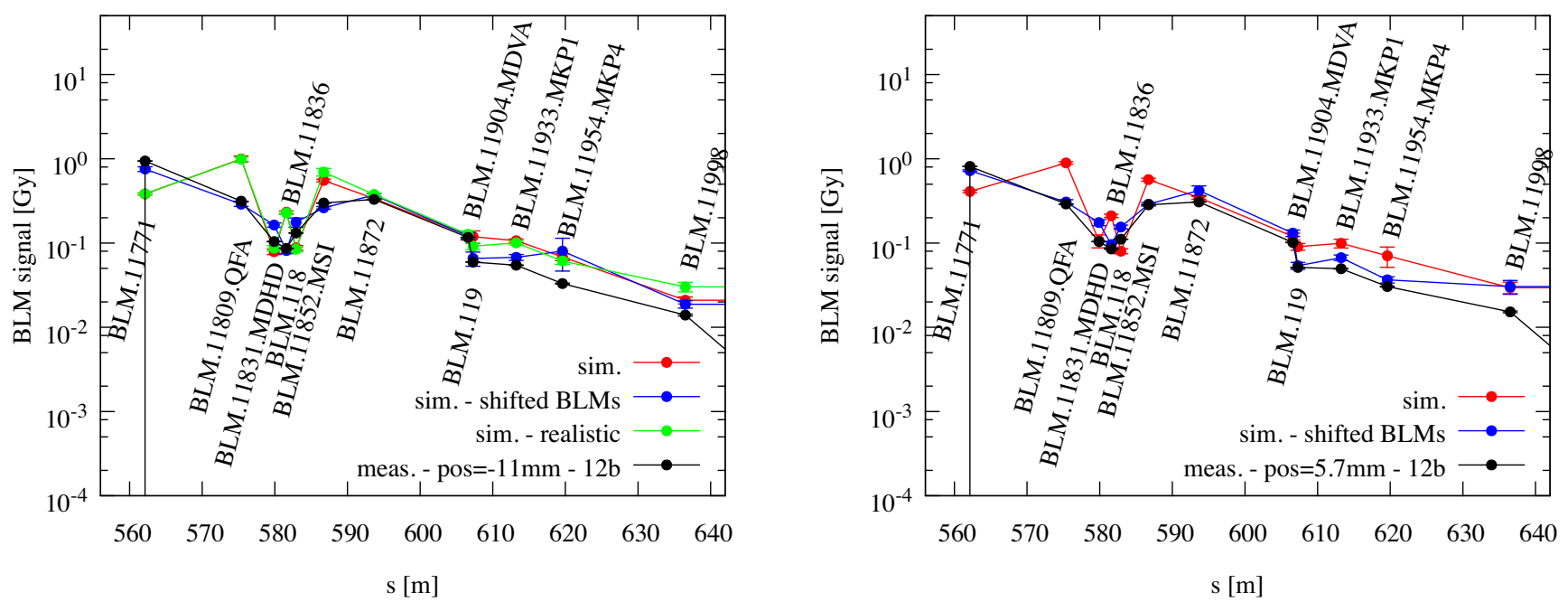

FIG. 18. Comparison between measured BLM signals (black curves) and predictions by simulations (colored curves) in LSS1, downstream of the scrapers, in case of full beam scraping with the horizontal (left frame) and the vertical (right frame) blades. Measurements with low-intensity beams are shown (see Fig. 11). The red curves show the simulation results with BLMs in their nominal positions, whereas the blue ones show the effect of a transverse displacement of the monitors in a more favorable position. The green curve shows simulation results in case more realistic assumptions are taken into account, i.e., with a broader beam distribution, the blade moving at the slower speed of $60 \mathrm{~mm} \mathrm{~s}^{-1}$ and tilted by $4^{\circ}$ about the longitudinal axis; simulations were carried out for the horizontal blade only. When visible, the error bars on the measurements refer to the LSB, whereas those on the simulated data refer to the statistical uncertainty only. 
In order to have an idea of the sensitivity of BLM signals on monitor positions, the blue curve in Fig. 18 shows simulation results in case BLMs were provocatively displaced in positions more favorable to the estimation of signals. Displacements are of the order of the transverse outer dimension of the monitor; only the two BLMs at the center of the plots (i.e., BLM.11872 and BLM.11904) were not moved, since the agreement is already remarkably good. For most of the BLMs upstream of BLM.11908 included (i.e., the one at the main beam dump), the agreement becomes extremely good, especially for those closest to the scrapers; just a couple of monitors give results off the measurements on the opposite side with respect to those at the reference position. The downstream BLMs get improved either, even though they suffer from statistics.

The green curve in the upper frame of Fig. 18 shows the effect on simulated BLM readouts in the case of more realistic assumptions, i.e., a broader beam distribution, the blade moving at the slower speed of $60 \mathrm{~mm} \mathrm{~s}^{-1}$ and tilted by $4^{\circ}$ about the longitudinal axis. Marginal variations can be seen, also on the BLMs downstream of the main beam dump. This proves that, even with quite important changes able to significantly affect the BCT signals (see Fig. 13), beam impact conditions at the scraper blades are not particularly relevant for BLM readouts, confirming the stability of BLM signals on actual scraping conditions and the reliability of the estimation of the total number of scraped protons.

\section{CONCLUSIONS}

The SPS scrapers are a multiturn cleaning system installed in the SPS aimed at halo removal before injection in the LHC; they consist of graphite blades swept through the beam immediately before beam transfer. Due to sweeping, the energy deposition is concentrated in the very first layers of the blade material directly impacted by the beam; if a too high amount of beam is scraped, such a high concentration can lead to damaging the blades.

A test with beam was carried out at the end of the SPS activity in 2013 to probe the endurance of the blades; the worst conditions of scraping were emulated. Even though the beam was prematurely dumped, the blades were damaged, producing a large local sublimation of graphite and inducing vacuum spikes covering some orders of magnitude. Moreover, the blade porosity was estimated on the basis of SEM images taken after dismounting the blades to $30 \%$ in the most damaged region, whereas levels of $\sim 2 \%$ were found in the pristine material. After the test, no substantial loss of performance of the blades for regular scraping of beam tails was visible.

The conditions of the test were reproduced in simulations by means of the FLUKA-SixTrack coupling, a simulation setup that allows to combine the refined physics models of the two codes for obtaining most accurate results. The energy deposition in the blades reached during the test reconstructed by simulations is well above the energy required to locally sublimate graphite, confirming the results of the SEM analyses.

The endurance test of the SPS scrapers also offered the occasion for an extensive benchmark of the simulation tool. The benchmark includes quantitative comparisons against beam intensity time profiles as measured by the BCT and losses recorded by BLMs. The quantitative benchmark against $\mathrm{BCT}$ readouts allowed to reconstruct tilt angle and speed of the blades during the test. The quantitative benchmark against BLM signals allowed to accurately estimate the number of scraped protons, an essential ingredient for the estimation of the energy deposition reached during the test.

The present work on the SPS scrapers is the first extensive study performed with the FLUKA-SixTrack coupling. The remarkable agreement between simulation results and measurements proves the maturity of the simulation tool and its readiness for other study cases.

\section{ACKNOWLEDGMENTS}

The authors would like to express their gratitude to V. Kain, R. Losito, M. Meddahi, F. L. Maciariello, and S. Cettur Cavè for very useful discussions, to Y. Papaphilippou and H. Bartosik for unveiling the secrets of the SPS optics, and to O. Mete and K. Cornelis for making the endurance test real.

[1] T. E. S. Group, 2020 Update of the European Strategy for Particle Physics (CERN, Geneva, 2020).

[2] Proceedings of the 61st ICFA Advanced Beam Dynamics Workshop on High-Intensity and High-Brightness Hadron Beams (HB2018), Daejeon, Korea, 2018, edited by D.-O. Jeon, D.-E. Kim, and V.R. Schaa (JACoW, Geneva, Switzerland, 2018).

[3] Proceedings of the 2014 Joint International Accelerator School: Beam Loss and Accelerator Protection, Newport Beach, CA, USA, 2014, edited by R. Schmidt, CERN Yellow Reports: School Proceedings (CERN, Geneva, 2016).

[4] O. Brüning, P. Collier, P. Lebrun, S. Myers, R. Ostojic, J. Poole, and P. Proudlock, LHC design report-Vol. 1, Report No. CERN-2004-003-V-1, CERN, Geneva, 2004, https://doi.org/10.5170/CERN-2004-003-V-1.

[5] A quench is the sudden transition of the magnet from the superconducting state to the normal conducting one.

[6] M. Benedikt, P. Collier, V. Mertens, J. Poole, and K. Schindl, LHC design report-Vol. 3, Report No. CERN2004-003-V-3, CERN, Geneva, 2004, https://doi.org/ 10.5170/CERN-2004-003-V-3.

[7] H. Burkhardt and R. Schmidt, Intensity and luminosity after beam scraping, Technical Report No. CERN-AB2004-032-ABP, CERN, Geneva, 2004, http://cds.cern.ch/ record/777311/files/ab-2004-032.pdf. 
[8] G. Arduini and H. Burkhardt, Transverse tail scraping in the SPS for clean LHC injection, Technical Report No. EDMS document 772782, CERN, Geneva, 2006.

[9] CERN, The $300 \mathrm{GeV}$ program, Report No. CERN-1050-E, CERN, Geneva, 1972, https://cds.cern.ch/record/104068? $\ln =\mathrm{en}$.

[10] V. Vlachoudis et al., FLUKA coupling to Sixtrack, in Proceedings of the ICFA Mini-Workshop on Tracking for Collimation in Particle Accelerators (CERN, Geneva, Switzerland, 2018), https://cds.cern.ch/record/2646800? $\ln =$ en.

[11] GIT repository of the FLUKA-sixTrack coupling code (CERN credentials required): https://gitlab.cern.ch/bmi/ fedb/coupling/fluka_coupling.

[12] F. Schmidt, sixTrack version 4.2.16: Single particle tracking code treating transverse motion with synchrotron oscillations in a symplectic manner; User's reference manual, Technical Reports No. CERN-SL-94-56 and No. CERN-SL-94-56-AP, CERN, Geneva, 1994, http:// cds.cern.ch/record/269474.

[13] sixTrack website: http://sixtrack.web.cern.ch/SixTrack.

[14] T. T. Böhlen, F. Cerutti, M. P. W. Chin, A. Fassò, A. Ferrari, P. G. Ortega, A. Mairani, P. R. Sala, G. Smirnov, and V. Vlachoudis, The FLUKA code: Developments and challenges for high energy and medical applications, Nucl. Data Sheets 120, 211 (2014).

[15] G. Battistoni et al., Overview of the FLUKA code, Ann. Nucl. Energy 82, 10 (2015).

[16] L. Drøsdal et al., SPS scraping and LHC transverse tails, in Proceedings of IPAC13, Shanghai, China, 2013 (2013), MOPWO032, http://accelconf.web.cern.ch/AccelConf/ IPAC2013/papers/mopwo032.pdf.

[17] M. Stockner and C. Fabjan, Beam loss calibration studies for high energy proton accelerators, Ph.D. thesis,
University of Technology, Vienna, 2007, presented on 27th Nov 2007.

[18] C. Roderick, L. Burdzanowski, and G. Kruk, The CERN accelerator logging service-10 Years in operation: A look at the past, present and future, Technical Report No. CERN-ACC-2013-0230, 2013.

[19] F. Léaux and G. A. Izquierdo, Microscopic observations of irradiated graphite scraper blades, Technical Report No. EDMS document 1339153, CERN, Geneva, 2014.

[20] 2nd ICFA Workshop on Machine Learning for Charged Particle Accelerators (PSI, Villigen, Switzerland, 2019), https://indico.psi.ch/event/6698.

[21] A. Edelen et al., Opportunities in machine learning for particle accelerators, arXiv:1811.03172.

[22] T. Kramer, G. Arduini, O. Berrig, E. Carlier, L. Ducimetiere, B. Goddard, A. Koschik, and J. Uythoven, Studies of beam losses from failures of SPS beam dump kickers, in Proceedings of the IEEE Particle Accelerator Conference (PAC), 2007, Albuquerque, USA, edited by C. Petit-Jean-Genaz (2007), pp. 1607-1609.

[23] J. Moyroud and R. Folch, Cumul de Jeux-Angle de la Lame Graphite, Technical Report No. EDMS document 1454838, CERN, Geneva, 2014.

[24] It should be kept in mind that no BLMs are installed in LSS3 (see Table II), but the ones at the regular FODO cells.

[25] V. Vlachoudis, FLAIR: A powerful but user friendly graphical interface for FLUKA, in Proceedings of International Conference on Mathematics, Computational Methods and Reactor Physics (M\&C 2009) (Saratoga Springs, New York, USA 2009), https://flair.web.cern.ch/ flair/doc/Flair_MC2009.pdf.

[26] CERN Drawing Directory-website: http://edms-service .web.cern.ch/edms-service/CDD. 